\title{
Impact of Sarvanga Ruksha Svedana on Physiological, Hematological and Biochemical Parameters in Young Healthy Subjects: An Exploratory Study
}

\author{
Saurabh Yadav ${ }^{1}$, Vandana Verma ${ }^{1 *}$ and Abinav ${ }^{2}$ \\ ${ }^{1}$ Department of Kriya Sharir, Faculty of Ayurveda, IMS, Banaras Hindu University, Varanasi - 221005, Uttar \\ Pradesh, India; vandana.verma04@gmail.com \\ 2Department of Kaya Chikitsa, Panchakarma Unit, Faculty of Ayurveda, IMS, Banaras Hindu University, \\ Varanasi - 221005, Uttar Pradesh, India
}

\begin{abstract}
Introduction: Svedana Karma (Sudation) is a therapeutic procedure used to pacify Vata, Kapha Dosha and also helps in detoxification of the body. Various methods have been described to induce sweating as per the disorder and the type of Dosha involved in pathogenesis. Sarvanga Ruksha svedana used in the present study is a type of Sagni Sveda (Kuti Sveda) in which heat is applied without prior Abhyanga (massage with oil). Objectives: To analyze certain physiological, haematological and biochemical parameters in blood and sweat and also to understand the mechanism of effects produced by Sarvanga Ruksha Svedan. Methods: An exploratory study was conducted on 30 clinically healthy subjects in the age group of 20-30 years. They were registered for the study after their written consent for Svedan procedure. The impact of Svedan was observed in terms of subject's pulse rate (PR), blood pressure (BP), Temperature and biochemical parameters like complete blood count (CBC), and renal function test (RFT), lipid profile, sweat biochemical analysis. Assessment of Prakriti was done as per the predominance of Dosha. Results: The changes observed in the BP, PR, temperature, hemoglobin (Hb), CBC, biochemical parameters such as serum and sweat electrolytes, blood lipid levels, serum urea, creatinine have shown significant effect of Sarvanga Ruksh Svedan. The difference in responses has been found to vary as per Prakriti also. Conclusion: Present study has shown several interesting findings leading to scientific interpretation of Svedan Karma and physiology of Svedavaha Srotas and also has provided a lead for future research in this area.
\end{abstract}

Keywords: Svedana, Passive Heat Therapy, Panchakarma, Sweat, Prakriti

\section{Introduction}

Svedana Karma (procedure to induce sweating) is one of the important treatment modality used by Ayurveda physician either as an independent intervention (Pradhankarma), or as Purvakarma or Paschatkarma of Panchkarma therapy. Ayurvedic Svedana Karma has similar systemic hemodynamic effects as produced after passive heat therapy. Various methods have been described to induce sweating as per the disorder and the type of Dosha involved in pathogenesis. Out of

${ }^{*}$ Author for correspondence

Article Received on: 16.01.2020

Revised on: 31.08.2020

two basic methods of Svedana, Niragni represents indirect method of body heating (warm without direct heat) examples of Niragni Svedana measures are heavy clothing, exercise etc. (Ch.Su.13/) ${ }^{1}$. The Saagni Sveda, includes dry or moist heat directly upon the body by various methods to heat it. Thus Niragni and Sagni Sveda can be considered as active \& passive heat therapies respectively. It produces various beneficial effects like liquefying the deposited Dosha, regulates Vata Dosha, helps in removal and pacification of Dosha, augments metabolism and appetite, softness and shines the skin, 
clears the channels, removes drowsiness, improves joint motility, flexibility in body parts, $(\mathrm{Ch} . \mathrm{Su} .14 / 67)^{1}$ as well as removal of waste metabolites in the form of sweat through Svedavaha Srotas (C.Vi.5/7-8) ${ }^{1}$.

The different method of Svedana Karma produces different kinds of cellular and hemodynamic effects which could be studied on scientific parameters. Heat applied in Svedana produces heat stress and initiates the thermoregulatory mechanism. It also produces various hemodynamic changes that help in excretion of metabolic wastes and enhanced immune mechanism. There are various types of Sarvanga Sveda which is described in Ayurveda compendia, an exploratory study was conducted to understand the effects of Sarvanga Ruksha Svedana therapy on clinically healthy subjects to analyze the compositions of sweat obtained during Svedan Karma and their physiological, hematological and biochemical changes after Svedana Karma procedure. Further, an attempt was made to observe the difference in responses to different Prakriti individuals.

\section{Material and Methods}

\subsection{Study Design}

The exploratory observational study was approved from the ethical committee of the institute (Dean/2015-16/ EC/148 dated 26.05.2016).

\subsection{Sampling Technique}

Purposive sampling was employed for selection of subjects.

\subsection{Study Population}

The sample was drawn from the student community of the institute purusing BAMS courses, during November to December in year 2017. Volunteers who were willing to be part of study were explained about the Svedan procedure and investigations. 30 clinically healthy subjects belonging to 20-30 years of age group as per inclusion and exclusion criteria were registered after obtaining their written consent for the procedure, out of which two subjects were the drop outs. All participants were healthy, non-smokers, non-alcoholic with no history of cardiovascular, hematological or metabolic disorders and not belonging to all Aswedya persons described by various scholar of Ayurveda (Ch. Su.14/16-19) ${ }^{1}$. Assessment of Prakriti was done by using proforma designed by Verma V et al. (2009) ${ }^{2}$. On the basis of predominance of Dosha all subjects were assigned as Vata, Pitta and Kapha Prakriti. Finally, 9, 11 and 8 individuals were assigned as Vata, Pitta and Kapha Prakriti respectively.

\subsection{Procedure of Sarvanga Ruksha Sveda}

All the subjects were given Ruksha sarvang Svedan (without prior Snehan Karma) after breakfast for three days in the morning between 8AM to 10AM. The duration of Svedan Karma per day approx.15-30 minutes and subjected to the temperature tolerance of the subject. It was performed in Sarvanga Sveda chamber of the hospital. Sarvang Ruksha Svedan apparatus constituted a wooden insulated chamber of measurement approximately $(\mathrm{L} \times \mathrm{W} \times \mathrm{D}$ $=47.5 \times 47.5 \times 46.5$ Inches). The chamber contained $\mathrm{a}$ retractable lid and a chair is placed inside the chamber, where the subjects was asked to sit with head-out position from chamber. The inner chamber consisted of 48 bulbs of 100 watt arranged symmetrically on the entire four walls. During the process, the subject's head was covered with a small wet towel. The patients who had undergone Sarvang Ruksha Svedan were allowed to change and rest in an enclosed area (without a direct air exposure) for few minutes before they could leave.

\subsection{Collection of Samples}

Subject's Pulse Rate (PR), Systolic Blood Pressure (SBP) and Diastolic Blood Pressure (DBP) were recorded by using sphygmomanometer, oral temperature was recorded by using clinical thermometer before the procedure and after coming out of the chamber. Venous blood sample (from cubital fossa) was taken on first day before and on third day after the completion of Svedan Karma for assessing lipid Profile, CBC (Complete Blood Count), and RFT (Renal Function Test). For the purpose of collecting sweat, subjects were instructed about the sweat collection procedure and provided with the sterile vial (marked in milliliter) for sweat collection. The individuals collected the sweat by putting vial in their arm pit. The collection of sweat sample started five minute after the subject has entered 
the chamber up to next ten minutes from the arm pit. Sweat sample was collected and analyzed on first and third day to access the variation in concentration of sodium, potassium, chloride ions, urea, and creatinine. All the hematological and biochemical (blood and sweat) parameters were analyzed in CCI lab of the hospital in the institute (Figure 1).

\subsection{Statistical Analysis}

Data analysis was done by IBM Statistical Package for Social Sciences (SPSS) Software Version 20. After

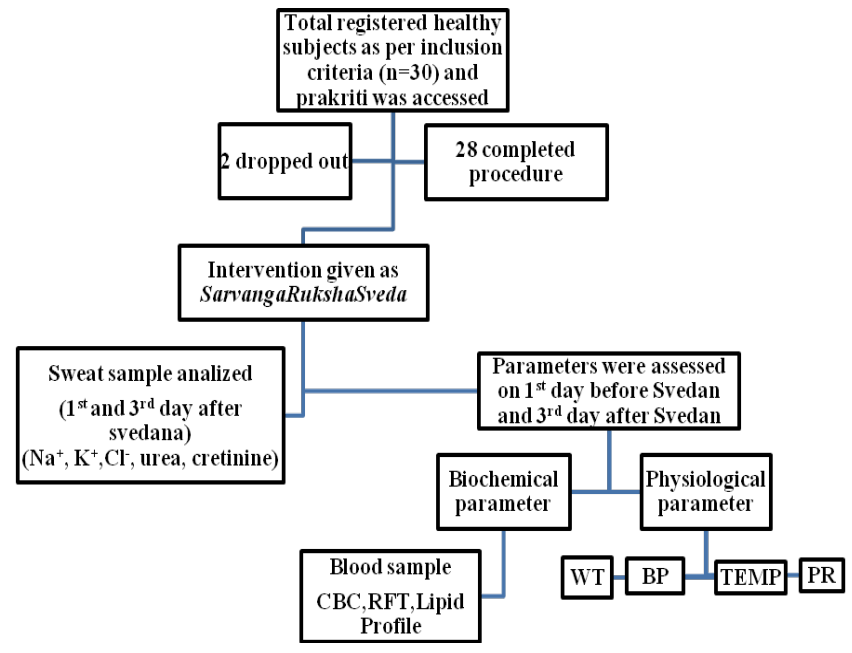

Figure 1. Schematic presentation of material and method. tabulation of data, appropriate statistical tests viz. cross tabulation, frequency, descriptive mean, and paired sample $t$ test, unpaired $t$ test, one-way ANOVA and Post Hoc Test were applied. P value of less than 0.05 was considered as statistically significant.

\section{Observation and Results}

The mean age of subjects was $22.93 \pm 2.100$. The mean height, weight and BMI of healthy subjects were $162.47 \pm 7.352,60.460 \pm 9.856$ and $22.30 \pm 3.313$ respectively.

The mean value of SBP and DBP on $1^{\text {st }}$ and $3^{\text {rd }}$ day before and after Sarvanga Ruksha Svedan was decreased. On comparing the mean of SBP on first day before and after third day through paired $t$ test, a significant decrease in SBP was found but an unsignificant decrease in DBP was also found. The mean value of pulse rate and temperature were found to be significantly increased. The mean of pulse rate and temperature on first and $3^{\text {rd }}$ day before and after Sarvanga Ruksha Svedan were increased. On comparing the first day before and after Svedan third day through paired $\mathrm{t}$ test, it was found to be significant (Table 1).

The mean value of red blood cells was not found to vary significantly before and after Svedan. The mean value of total leukocyte counts and neutrophil percentage increased. On applying paired $t$ test, the change was found to be significant in neutrophil

Table 1. Showing the Mean \pm SD of physiological parameters before and after Sarvanga Ruksha Svedan

\begin{tabular}{|c|c|c|c|c|c|c|c|}
\hline \multirow{2}{*}{ Variables } & \multicolumn{4}{|c|}{ MEAN $\pm S D$} & \multicolumn{3}{|c|}{ Within the group comparison (paired t-test) } \\
\hline & BS1 & AS1 & BS3 & AS3 & BS1 v/s AS1 & BS3 v/s AS3 & $\mathrm{BS} 1 \mathrm{v} / \mathrm{s}$ AS3 \\
\hline \multirow{2}{*}{$\mathrm{SBP}(\mathrm{mmHg})$} & $116.53 \pm$ & $114.00 \pm$ & $116.64 \pm$ & $113.93 \pm$ & $t=2.59$ & $t=2.572$ & $\mathrm{t}=2.880$ \\
\hline & 10.061 & 9.642 & 7.405 & 9.970 & $p=0.01$ & $p=0.016$ & $p=0.008$ \\
\hline \multirow{2}{*}{$\mathrm{DBP}(\mathrm{mmHg})$} & $78.27 \pm$ & $77.67 \pm$ & $78.64 \pm$ & $78.29 \pm$ & $t=0.752$ & $t=0.586$ & $t=0.487$ \\
\hline & 7.891 & 6.850 & 4.147 & 5.649 & $p=0.458$ & $p=0.562$ & $p=0.630$ \\
\hline \multirow{2}{*}{ PR(per min) } & $76.23 \pm$ & $98.73 \pm$ & $75.71 \pm$ & $95.96 \pm$ & $t=12.992$ & $\mathrm{t}=13.428$ & $t=9.866$ \\
\hline & 6.296 & 12.387 & 6.127 & 11.348 & $\mathrm{p}<0.001$ & $\mathrm{p}<0.001$ & $p=0.001$ \\
\hline \multirow{2}{*}{$\operatorname{TEMP}\left({ }^{\circ} \mathrm{C}\right)$} & $36.63 \pm$ & $37.35 \pm$ & $36.61 \pm$ & $37.32 \pm$ & $t=6.134$ & $t=8.337$ & $t=8.647$ \\
\hline & 0.435 & 0.579 & 0.492 & 0.373 & $p<0.001$ & $p<0.001$ & $p=0.001$ \\
\hline
\end{tabular}

BS1- (Before Sarvanga Ruksha Svedan day 1), AS1-(After Sarvanga Ruksha Svedan day 1), BS3-(Before Sarvanga Ruksha Svedan day 3), AS3-(After Sarvanga Ruksha Svedan day 3) 
percentage. The mean values of other different leukocytes (eosinophil, basophil, lymphocyte, and monocyte) decreased but were not found to be significant. The mean value of hemoglobin, $\mathrm{MCV}$, $\mathrm{MCH}, \mathrm{MCHC}, \mathrm{MPV}$ increased whereas platelet, HCT decreased but on applying paired $t$ test the changes were not found to be significant (Table 2).
The mean value of serum sodium, chloride and urea concentration were decreased whereas potassium, creatinine concentration was increased after Sarvanga Ruksha Svedan but on applying paired t test the serum sodium, chloride concentration changes were found to be significant. The mean value of cholesterol, high density lipoprotein, low density lipoprotein

Table 2. Showing the Mean \pm SD of hematological parameters before and after Sarvanga Ruksha Svedan

\begin{tabular}{cccc}
\hline \multirow{2}{*}{ Variables } & \multicolumn{2}{c}{ MEAN \pm SD } & \multirow{2}{*}{ Within group comparison Paired t-test } \\
\cline { 2 - 3 } & $4.62 \pm 0.72$ & $4.62 \pm 0.64$ & $\mathrm{t}=0.296, \mathrm{p}=0.769$ \\
RBC(million/mm $\mathrm{mm}^{3}$ ) & $7.41 \pm 1.53$ & $7.45 \pm 0.97$ & $\mathrm{t}=0.166, \mathrm{p}=0.869$ \\
WBC(per mm $\left.{ }^{3}\right)$ & $57.11 \pm 14.08$ & $59.86 \pm 13.81$ & $\mathrm{t}=2.693, \mathrm{p}=\mathbf{0 . 0 1 2}$ \\
NEUTROPHIL (\%) & $30.02 \pm 8.43$ & $28.54 \pm 5.90$ & $\mathrm{t}=1.045, \mathrm{p}=0.305$ \\
LYMPHOCYTE (\%) & $7.08 \pm 10.10$ & $5.39 \pm 1.17$ & $\mathrm{t}=0.910, \mathrm{p}=0.371$ \\
MONOCYTE (\%) & $4.55 \pm 4.719$ & $3.98 \pm 4.906$ & ${ }^{*} \mathrm{z}=2.037, \mathrm{p}=0.052$ \\
EOSINOPHIL (\%) & $0.43 \pm 0.300$ & $0.40 \pm 0.203$ & $\mathrm{t}=0.372, \mathrm{p}=0.713$ \\
BASOPHIL (\%) & $262.93 \pm 72.51$ & $258.61 \pm 66.62$ & $\mathrm{t}=1.141, \mathrm{p}=0.264$ \\
PLATELET(x10 $/$ (cumm) & $11.20 \pm 1.325$ & $11.29 \pm 13.09$ & $\mathrm{t}=0.297, \mathrm{p}=0.769$ \\
MPV & $13.44 \pm 1.594$ & $13.47 \pm 1.73768$ & $\mathrm{t}=0.961, \mathrm{p}=0.345$ \\
HB(gm/dl) & $41.76 \pm 4.354$ & $41.43 \pm 4.797$ & $\mathrm{t}=1.084, \mathrm{p}=0.288$ \\
HCT (\%) & $89.08 \pm 19.005$ & $92.31 \pm 9.67795$ & $\mathrm{t}=0.986, \mathrm{p}=0.333$ \\
MCV (femtoliters) & $29.56 \pm 4.06$ & $29.80 \pm 3.61$ & $\mathrm{t}=0.273, \mathrm{p}=0.787$ \\
MCH (picograms) & $31.99 \pm 0.858$ & $32.10 \pm 1.11$ & $\mathrm{t}=1.652, \mathrm{p}=0.110$ \\
MCHC (\%) & & &
\end{tabular}

${ }^{*}$ Non parametric test

was increased but triglycerides was decreased after application of Sarvang Svedan, but the changes were not found to be significant (Table 3).

The mean value of sweat total protein and albumin decreased after Sarvanga Ruksha Svedana and the mean value of sweat urea, creatinine, and sodium, chloride concentration was increased; potassium concentration was decreased in sweat after Svedan procedure. On applying paired $\mathrm{t}$ test, change in sodium concentration was found to be significant (Table 4).

The mean value of duration of tolerance to heat in Sarvanga Ruksha Svedan in Vata, Pitta, Kapha Prakriti individuals on day first and third was 25.78 \pm 5.826 , $26.89 \pm 5.442, \quad 24.00 \pm 4.714$ and $25.80 \pm 4.709$, $28.13 \pm 3.137,28.88 \pm 3.523$ respectively. The changes were not found to be statistically significant in paired $t$ test of Vata and Kakha Prakriti while in Pitta Prakriti it was found to be significant ( $\mathrm{p}=0.001$ ) (Table 5).
The mean values of SBP decreased in all Prakriti individuals but it was significant for Pitta Prakriti individuals. In one-way ANOVA was not found to vary significantly (Table 6).

The mean value of DBP decreased in Vata and Pitta Prakriti subjects but increased in Kapha Prakriti subjects on both first and third day after Svedan. On applying paired $t$ test the difference was not found to vary significantly. In One Way ANOVA it was observed that the difference in mean values of diastolic blood pressure was significant for $B S 3(p=0.030)$ but on applying Post Hoc Test no significant pairs were found for $\mathrm{V}$ vs $\mathrm{K}$ $(\mathrm{p}=0.057)$ (Table 7).

The mean value of pulse rate increased in all Prakriti subjects on both days after applying Sarvanga Ruksha Svedana. It was found to vary significantly on applying paired t test in all Prakriti individuals $(\mathrm{p}<0.001)$. On comparing the mean value of pulse rate as per Prakriti 
Table 3. Showing the Mean \pm SD of biochemical parameters in blood before and after Sarvanga Ruksha Svedan

\begin{tabular}{|c|c|c|c|}
\hline \multirow{2}{*}{ Variables } & \multicolumn{2}{|c|}{$\mathrm{MEAN} \pm \mathrm{SD}$} & \multirow{2}{*}{$\begin{array}{c}\text { Within group comparison Pairec } \\
\text { t-test }\end{array}$} \\
\hline & BS1 & AS3 & \\
\hline SODIUM(mmol//l) & $141.11 \pm 2.55$ & $140.51 \pm 2.15$ & $t=2.17, p=0.04$ \\
\hline POTASSIUM(mmol//l) & $4.27 \pm 0.41$ & $4.40 \pm 0.36$ & $t=0.57, p=0.57$ \\
\hline CHLORIDE(mmol//l) & $103.17 \pm 2.53$ & $102.35 \pm 2.67$ & $t=2.15, p=0.04$ \\
\hline $\mathrm{CR}(\mathrm{mg} / \mathrm{dl})$ & $0.84 \pm 0.17$ & $1.57 \pm 0.23$ & $t=1.04, p=0.30$ \\
\hline $\operatorname{UREA}(\mathrm{mg} / \mathrm{dl})$ & $26.13 \pm 6.42$ & $24.84 \pm 7.74$ & $t=1.17, p=0.24$ \\
\hline CHOLESTROL $(\mathrm{mg} / \mathrm{dl})$ & $168.67 \pm 442.25$ & $169.54 \pm 38.61$ & $\mathrm{t}=0.62, \mathrm{p}=0.54$ \\
\hline $\mathrm{HDL}(\mathrm{mg} / \mathrm{dl})$ & $50.69 \pm 10.77$ & $51.38 \pm 11.82$ & $\mathrm{t}=0.18, \mathrm{p}=0.85$ \\
\hline $\mathrm{LDL}(\mathrm{mg} / \mathrm{dl})$ & $98.58 \pm 37.182$ & $99.64 \pm 35.787$ & $\mathrm{t}=0.589, \mathrm{p}=0.561$ \\
\hline $\mathrm{TG}(\mathrm{mg} / \mathrm{dl})$ & $133.17 \pm 50.519$ & $122.35 \pm 43.105$ & $\mathrm{t}=1.995, \mathrm{p}=0.056$ \\
\hline
\end{tabular}

Table 4. Showing the Mean \pm SD of sweat biochemical parameters before and after Sarvanga Ruksha Svedan

\begin{tabular}{cccc}
\hline Variables of sweat Analysis & \multicolumn{2}{c}{ MEAN \pm SD } & $\begin{array}{c}\text { Within group comparison } \\
\text { (Non-parametric test) }\end{array}$ \\
\cline { 2 - 3 } Protein $(\mathrm{gm} / \mathrm{dl})$ & $0.23 \pm 0.16$ & $0.14 \pm 0.10$ & $\mathrm{zS}=1.00, \mathrm{p}=0.32$ \\
Albumin $(\mathrm{gm} / \mathrm{dl})$ & $0.04 \pm 0.06$ & $0.02 \pm 0.049$ & $\mathrm{z}=0.001, \mathrm{p}=1.00$ \\
Creatinine $(\mathrm{mg} / \mathrm{dl})$ & $0.48 \pm 0.25$ & $0.49 \pm 0.24$ & $\mathrm{z}=0.57, \mathrm{p}=0.57$ \\
Urea $(\mathrm{mg} / \mathrm{dl})$ & $104.15 \pm 43.56$ & $106.74 \pm 34.40$ & ${ }^{*} \mathrm{t}=0.21, \mathrm{p}=0.83$ \\
Sodium $(\mathrm{mmol} / \mathrm{l})$ & $90.22 \pm 42.54$ & $102.93 \pm 43.71$ & ${ }^{*} \mathrm{t}=2.07, \mathrm{p}=0.04$ \\
Potassium $(\mathrm{mmol} / \mathrm{l})$ & $21.69 \pm 17.67$ & $18.49 \pm 6.62$ & $\mathrm{z}=1.07, \mathrm{p}=0.23$ \\
Chloride $(\mathrm{mmol} / \mathrm{l})$ & $75.20 \pm 31.92$ & $79.04 \pm 35.19$ & $\mathrm{z}=0.26, \mathrm{p}=0.79$ \\
\hline
\end{tabular}

${ }^{\star}$ Paired t-test

Table 5. Showing the Mean \pm SD of time duration of heat tolerance (in minute) in Sarvanga Ruksha Svedan as per Prakriti. $(\mathrm{n}=28)$

\begin{tabular}{|c|c|c|c|}
\hline \multirow{2}{*}{ Prakriti } & \multicolumn{2}{|c|}{$\mathrm{MEAN} \pm \mathrm{SD}$} & \multirow{2}{*}{ Within the group comparison (paired t-test) } \\
\hline & Day1 & Day3 & \\
\hline$V(n=9)$ & $25.78 \pm 5.82$ & $26.89 \pm 5.44$ & $\mathrm{t}=1.971, \mathrm{p}=0.084$ \\
\hline$P(n=11)$ & $24.00 \pm 4.71$ & $25.80 \pm 4.70$ & $\mathrm{t}=7.21, \mathrm{p}=0.001$ \\
\hline$K(n=8)$ & $28.13 \pm 3.13$ & $28.88 \pm 3.52$ & $\mathrm{t}=1.11, \mathrm{p}=0.30$ \\
\hline One Way ANOVA & $F=4.31, p=0.024$ & $F=5.28, p=0.012$ & \\
\hline Post Hoc Test & $\begin{array}{l}\text { V v/s P, p }=\mathbf{0 . 0 5} \\
\text { V v/s K, p }=1.00 \\
\text { P v/s K, p }=0.096\end{array}$ & $\begin{array}{c}\mathbf{V} \text { v/s P, p }=\mathbf{0 . 0 3 3} \\
V \mathrm{v} / \mathrm{s} \mathrm{K}, \mathrm{p}=1.00 \\
\mathbf{P} \mathbf{v} / \mathbf{s} \mathbf{K}, \mathbf{p}=\mathbf{0 . 0 4 5}\end{array}$ & \\
\hline
\end{tabular}


Impact of Sarvanga Ruksha Svedana on Physiological, Hematological and Biochemical Parameters in Young Healthy Subjects: An Exploratory Study

Table 6. Showing the Effect of Sarvanga Ruksha Svedan on Systolic blood pressure $(\mathrm{mmHg})$ as per Prakriti $(\mathrm{n}=28)$

\begin{tabular}{cccccc}
\hline & \multicolumn{3}{c}{ MEAN \pm SD } & & $\begin{array}{c}\text { Comparison within the } \\
\text { group(paired t-test) }\end{array}$ \\
\cline { 2 - 6 } Prakriti & BS1 & AS1 & BS3 & AS3 & BS1 v/s AS3 \\
\hline $\mathrm{n}(\mathrm{n}=9)$ & $115.56 \pm 9.63$ & $112.44 \pm 11.65$ & $114.89 \pm 6 . .49$ & $111.78 \pm 9.51$ & $\mathrm{t}=2.135, \mathrm{p}=0.065$ \\
$\mathrm{P}(\mathrm{n}=11)$ & $119.60 \pm 4.88$ & $115.60 \pm 6.17$ & $120.20 \pm 5.37$ & $117.00 \pm 5.67$ & $\mathrm{t}=3.354, \mathrm{p}=\mathbf{0 . 0 0 8}$ \\
$\mathrm{K}(\mathrm{n}=8)$ & $118.00 \pm 10.95$ & $115.00 \pm 11.56$ & $116.25 \pm 8.03$ & $115.25 \pm 12.8$ & $\mathrm{t}=1.925, \mathrm{p}=0.096$ \\
One Way & $\mathrm{F}=0.521$ & $\mathrm{~F}=0.262$ & $\mathrm{~F}=1.663$ & $\mathrm{~F}=0.772$ & \\
ANOVA & $\mathrm{p}=0.600$ & $\mathrm{p}=0.772$ & $\mathrm{p}=0.211$ & $\mathrm{p}=0.473$ & \\
Post hoc & - & - & - & - & \\
\hline
\end{tabular}

through One-way ANOVA varied significantly for BS3 $(p=0.004)$ and AS3 ( $p=0.028)$. The significant pair found through Post Hoc Test was P v/s K ( $\mathrm{p}=0.026$ for BS3 and $\mathrm{p}=0.003$ for AS3) (Table 8).

The mean value of oral temperature increased on both days after applying Svedana in all Prakriti individuals. It was found to vary significantly on applying paired t test $(\mathrm{V}, \mathrm{p}=0.004 \mathrm{~K}, \mathrm{P}, \mathrm{p}=0.001)$. On intergroup comparison through One-way ANOVA, it was not found to vary significantly (Table 9).

The data shows that mean value of platelet concentration increased on day third in all Prakriti individuals. On applying paired t test it was found to be significant for Pitta Prakriti individuals ( $\mathrm{p}=0.019$ ). On applying One Way ANOVA it was found to vary significantly on BS1 $(p=0.024)$. The significant pair found through Post Hoc Test was V v/s P ( $\mathrm{p}=0.030)$ (Table 10)
The data shows that mean value of Neutrophil percentage increased on day third compared to day first in all Prakriti individuals. It was found statistically significant on applying paired t test for Pitta Prakriti individuals $(\mathrm{p}=0.049)$. However, it did not vary significantly as per Prakriti on applying One Way ANOVA (Table 11).

The data shows that mean value of serum chloride concentration decreased on day third as compared to day first in Vata Prakriti and Pitta Prakriti individuals while in Kapha Prakriti individuals it was increased. It was found statistically significant on applying paired $\mathrm{t}$ test for Pitta Prakriti individuals ( $\mathrm{p}=0.035$ ). On applying One Way ANOVA it was not found to vary significantly as per Prakriti (Table 12).

The data shows that the mean value of sweat creatinine concentration was increased on day third as compared to day first in all Prakriti individuals. It

Table 7. Showing the Effect of Sarvanga Ruksha Svedan therapy on Diastolic blood pressure $(\mathrm{mmHg})$ as per Prakriti

\begin{tabular}{|c|c|c|c|c|c|}
\hline \multirow{2}{*}{ Prakriti } & \multicolumn{4}{|c|}{$\mathrm{MEAN} \pm \mathrm{SD}$} & \multirow{2}{*}{$\begin{array}{l}\text { Within the group comparison (paired t-test) } \\
\text { BS1 v/s AS3 }\end{array}$} \\
\hline & BS1 & AS1 & BS3 & AS3 & \\
\hline$V(n=9)$ & $\begin{array}{c}79.11 \pm \\
8.78\end{array}$ & $\begin{array}{c}76.44 \pm \\
7.66\end{array}$ & $\begin{array}{c}78.00 \pm \\
4.69\end{array}$ & $\begin{array}{c}77.33 \pm \\
3.74\end{array}$ & $\begin{array}{l}t=0.81 \\
p=0.43\end{array}$ \\
\hline$P(n=11)$ & $\begin{array}{c}78.80 \pm \\
6.05\end{array}$ & $\begin{array}{c}79.20 \pm \\
6.61\end{array}$ & $\begin{array}{c}78.40 \pm \\
2.79\end{array}$ & $\begin{array}{c}77.40 \pm \\
3.27\end{array}$ & $\begin{array}{l}t=1.46 \\
p=0.17\end{array}$ \\
\hline$K(n=8)$ & $\begin{array}{c}81.00 \pm \\
5.01\end{array}$ & $\begin{array}{c}79.50 \pm \\
3.81\end{array}$ & $\begin{array}{c}80.75 \pm \\
3.84\end{array}$ & $\begin{array}{c}82.50 \pm \\
5.73\end{array}$ & $\begin{array}{l}t=1.36 \\
p=0.21\end{array}$ \\
\hline $\begin{array}{c}\text { One Way } \\
\text { ANOVA }\end{array}$ & $\begin{array}{l}F=0.25 \\
p=0.77\end{array}$ & $\begin{array}{l}F=0.62 \\
p=0.54\end{array}$ & $\begin{array}{l}F=1.27 \\
p=0.29\end{array}$ & $\begin{array}{l}F=4.05 \\
p=0.03\end{array}$ & \\
\hline Post Hoc Test & - & - & - & $\begin{array}{l}V \mathrm{v} / \mathrm{s} P \mathrm{p}=1.00 \\
\mathrm{~V} \mathrm{v} / \mathrm{s} \mathrm{K} 0.061 \\
\mathrm{P} \mathrm{v} / \mathrm{s} \mathrm{K} 0.057\end{array}$ & \\
\hline
\end{tabular}


Table 8. Showing the Effect of Sarvanga Ruksha Svedan on pulse rate (in minute) as per Prakriti

\begin{tabular}{|c|c|c|c|c|c|}
\hline \multirow{2}{*}{ Prakriti } & \multicolumn{4}{|c|}{$\mathrm{MEAN} \pm \mathrm{SD}$} & \multirow{2}{*}{$\begin{array}{c}\text { Within the group comparison (paired t-test } \\
\text { BS1 v/s AS3 }\end{array}$} \\
\hline & BS1 & AS1 & BS3 & AS3 & \\
\hline \multirow{2}{*}{$V(n-9)$} & $75.11 \pm$ & $101.78 \pm$ & $76.00 \pm$ & $95.44 \pm$ & $\mathrm{t}=8.352$ \\
\hline & 6.93 & 15.17 & 4.12 & 8.01 & $\mathrm{p}<0.001$ \\
\hline \multirow{2}{*}{$P(n-11)$} & $78.00 \pm$ & $96.20 \pm$ & $79.00 \pm$ & $99.40 \pm$ & $t=10.846$ \\
\hline & 6.12 & 9.44 & 5.98 & 9.04 & $\mathrm{p}<0.001$ \\
\hline \multirow{2}{*}{$K(n-8)$} & $74.75 \pm$ & $94.00 \pm$ & $70.25 \pm$ & $88.00 \pm$ & $t=7.780$ \\
\hline & 6.84 & 11.46 & 4.46 & 7.92 & $p<0.001$ \\
\hline \multirow{3}{*}{$\begin{array}{c}\text { One Way } \\
\text { ANOVA }\end{array}$} & $\mathrm{F}=0.679$ & $\mathrm{~F}=0.940$ & $F=0.629$ & $F=4.152$ & \\
\hline & $p=0.516$ & $p=0.404$ & $p=0.004$ & $p=0.028$ & \\
\hline & & & $\mathrm{V} v / \mathrm{s} P \mathrm{p}=0.946$ & $\begin{array}{l}\mathrm{VV} / \mathrm{s} P \\
p=0.609\end{array}$ & \\
\hline \multirow[t]{2}{*}{ Post Hoc Test } & & & $\mathrm{V} v / \mathrm{s} \mathrm{K} \mathrm{p}=0.241$ & V v/s K 0.078 & \\
\hline & & & $\mathrm{P} \mathrm{v} / \mathrm{s} \mathrm{K} \mathrm{p=0.026}$ & $\begin{array}{c}\text { P v/s K } \\
0.003\end{array}$ & \\
\hline
\end{tabular}

Table 9. Showing the Effect of Sarvanga Ruksha Svedan on oral temperature (in ${ }^{0} \mathrm{C}$ ) as per Prakriti

\begin{tabular}{|c|c|c|c|c|c|}
\hline \multirow{2}{*}{ Prakriti } & \multicolumn{4}{|c|}{$\mathrm{MEAN} \pm \mathrm{SD}$} & \multirow{2}{*}{$\begin{array}{l}\text { Within the group comparison (paired } \\
\text { t-test) } \\
\text { BS1 v/s AS3 }\end{array}$} \\
\hline & BS1 & AS1 & BS3 & AS3 & \\
\hline$V(n=9)$ & $\begin{array}{c}36.22 \pm \\
0.44\end{array}$ & $\begin{array}{c}37.11 \pm \\
0.92\end{array}$ & $\begin{array}{c}36.67 \pm \\
0.50\end{array}$ & $\begin{array}{c}37.33 \pm \\
0.50\end{array}$ & $\begin{array}{l}t=4.000 \\
p=0.004\end{array}$ \\
\hline$P(n=11)$ & $\begin{array}{c}36.70 \pm \\
0.48\end{array}$ & $\begin{array}{c}37.40 \pm \\
0.51\end{array}$ & $\begin{array}{c}36.80 \pm \\
0.42\end{array}$ & $\begin{array}{c}37.50 \pm \\
0.52\end{array}$ & $\begin{array}{c}\mathrm{t}=4.583 \\
\mathrm{p}=\mathbf{0 . 0 0 1}\end{array}$ \\
\hline$K(n=8)$ & $\begin{array}{c}36.50 \pm \\
0.53\end{array}$ & $\begin{array}{c}37.13 \pm \\
0.64\end{array}$ & $\begin{array}{c}36.25 \pm \\
0.46\end{array}$ & $\begin{array}{c}37.13 \pm \\
0.64\end{array}$ & $\begin{array}{c}t=7.000 \\
p=\mathbf{0 . 0 0 1}\end{array}$ \\
\hline $\begin{array}{l}\text { One Way } \\
\text { ANOVA }\end{array}$ & $\begin{array}{l}F=3.194 \\
p=0.059\end{array}$ & $\begin{array}{l}F=0.418 \\
p=0.663\end{array}$ & $\begin{array}{l}F=1.423 \\
p=0.257\end{array}$ & $\begin{array}{l}F=1.220 \\
p=0.313\end{array}$ & \\
\hline Post Hoc Test & - & - & - & - & \\
\hline
\end{tabular}

Table 10. Showing the Effect of Sarvanga Ruksha Svedan on platelet count $\left(\times 10^{3} / \mathrm{cmm}\right)$ as per Prakriti

\begin{tabular}{|c|c|c|c|}
\hline \multirow{2}{*}{ Prakriti } & \multicolumn{2}{|c|}{$\mathrm{MEAN} \pm \mathrm{SD}$} & \multirow{2}{*}{ Within the group comparison (paired t-test) } \\
\hline & AS1 & AS3 & \\
\hline$V(n=9)$ & $224.89 \pm 36.50$ & $228.11 \pm 42.20$ & $\begin{array}{l}t=0.550 \\
p=0.598\end{array}$ \\
\hline$P(n=11)$ & $312.50 \pm 86.11$ & $327.60 \pm 75.05$ & $\begin{array}{c}t=2.843 \\
p=0.019\end{array}$ \\
\hline$K(n=8)$ & $243.00 \pm 69.76$ & $247.13 \pm 65.14$ & $\begin{array}{l}t=0.624 \\
p=0.553\end{array}$ \\
\hline One Way ANOVA & $\begin{array}{l}\mathrm{F}=4.389 \\
\mathrm{p}=0.024\end{array}$ & $\begin{array}{l}F=3.120 \\
p=0.062\end{array}$ & \\
\hline Post Hoc Test & $\begin{array}{l}\mathbf{V} \mathbf{v} / \mathbf{s} \mathbf{P} \mathbf{p}=\mathbf{0 . 0 3 0} \\
\mathrm{V} \mathbf{v} / \mathrm{s} K \mathrm{p}=1.000 \\
\mathrm{P} \mathrm{v} / \mathrm{s} K \mathrm{~K}=0.126\end{array}$ & - & \\
\hline
\end{tabular}


Impact of Sarvanga Ruksha Svedana on Physiological, Hematological and Biochemical Parameters in Young Healthy Subjects: An Exploratory Study

Table 11. Showing the Effect of Sarvanga Ruksha Svedan on Neutrophil (in percentage) as per Prakriti

\begin{tabular}{cccc}
\hline \multirow{2}{*}{ Prakriti } & \multicolumn{2}{c}{ MEAN \pm SD } & Within the group comparison (paired t-test) \\
\cline { 2 - 3 } $\mathrm{V}(\mathrm{n}=9)$ & $59.44 \pm 8.44$ & $62.33 \pm 5.85$ & $\mathrm{t}=1.364$ \\
$\mathrm{P}(\mathrm{n}=11)$ & $55.30 \pm 20.07$ & $59.40 \pm 20.71$ & $\mathrm{p}=0.210$ \\
& & & $\mathrm{t}=\mathbf{2 . 2 8 1}$ \\
$\mathrm{K}(\mathrm{n}=8)$ & $56.63 \pm 12.07$ & $57.63 \pm 11.31$ & $\mathrm{t}=0.579$ \\
& $\mathrm{~F}=0.193$ & $\mathrm{~F}=0.228$ & \\
One Way ANOVA & $\mathrm{p}=0.826$ & $\mathrm{p}=0.797$ & \\
Post Hoc Test & - & - & \\
\hline
\end{tabular}

was found to vary statistically significant on applying nonparametric test in Pitta Prakriti individuals $(\mathrm{p}=0.037)$. On applying One Way ANOVA sweat creatinine concentration did not vary significantly (Table 13).

The data shows that mean value of sweat sodium concentration increased on day third in all Prakriti individuals. It was found to be statistically significant on applying paired t test for Pitta Prakriti individuals $(\mathrm{p}=0.023)$. On applying One Way ANOVA, the mean value of sweat sodium concentration did not vary significantly as per Prakriti (Table 14).

The mean values of sweat chloride concentration decreased on day third in Vata and Kapha Prakriti

Table 12. Showing the Effect of Sarvanga Ruksha Svedan on serum Chloride ( $\mathrm{mmol} / \mathrm{l})$ as per Prakriti

\begin{tabular}{cccc}
\hline \multirow{2}{*}{ Prakriti } & \multicolumn{2}{c}{ MEAN \pm SD } & Within the group comparison (paired t-test) \\
\cline { 2 - 3 } $\mathrm{V}(\mathrm{n}=9)$ & $103.11 \pm 2.08$ & $\mathrm{AS3}$ & $\mathrm{t}=1.818$ \\
$\mathrm{P}(\mathrm{n}=11)$ & $103.50 \pm 1.95$ & $102.00 \pm 2.17$ & $\mathrm{p}=0.107$ \\
& & $\mathrm{t}=2.475$ & $\mathrm{p}=0.035$ \\
$\mathrm{~K}(\mathrm{n}=8)$ & $102.50 \pm 3.58$ & $103.00 \pm 4.00$ & $\mathrm{t}=0.592$ \\
& $\mathrm{~F}=0.324$ & $\mathrm{p}=0.573$ \\
One ay ANOVA & $\mathrm{p}=0.727$ & $\mathrm{p}=0.680$ & \\
Post Hoc Test & - & - & \\
\hline
\end{tabular}

Table 13. Showing the Effect of Sarvanga Ruksha Svedan on sweat creatinine (mg/dl) as per Prakriti

\begin{tabular}{|c|c|c|c|}
\hline \multirow{2}{*}{ Prakriti } & \multicolumn{2}{|c|}{$\mathrm{MEAN} \pm \mathrm{SD}$} & \multirow{2}{*}{ Within the group comparison (nonparametric test) } \\
\hline & AS1 & AS3 & \\
\hline$V(n=9)$ & $0.33 \pm 0.46$ & $0.37 \pm 0.50$ & $\begin{array}{l}z=0.000 \\
p=1.000\end{array}$ \\
\hline$P(n=11)$ & $0.20 \pm 0.42$ & $0.60 \pm 0.51$ & $\begin{array}{l}z=2.449 \\
p=0.037\end{array}$ \\
\hline$K(n=8)$ & $0.63 \pm 0.51$ & $0.68 \pm 0.51$ & $\begin{array}{l}z=1.000 \\
p=0.351\end{array}$ \\
\hline One Way ANOVA & $\begin{array}{l}F=0.688 \\
p=0.513\end{array}$ & $\begin{array}{l}F=0.873 \\
p=0.431\end{array}$ & \\
\hline Post Hoc Test & & & \\
\hline
\end{tabular}


Table 14. Showing the Effect of Sarvanga Ruksha Svedan on sweat sodium (mmol/l) as per Prakriti

\begin{tabular}{cccc}
\hline \multirow{2}{*}{ Prakriti } & \multicolumn{2}{c}{ MEAN \pm SD } & Within the group comparison (paired t-test) \\
\cline { 2 - 3 } $\mathrm{N}(\mathrm{n}=9)$ & $85.44 \pm 43.78$ & $92.22 \pm 35.82$ & $\mathrm{t}=0.593$ \\
& & $\mathrm{p}=0.569$ \\
$\mathrm{P}(\mathrm{n}=11)$ & $88.10 \pm 52.72$ & $121.00 \pm 51.51$ & $\mathrm{t}=2.730$ \\
& & $\mathrm{p}=\mathbf{0 . 0 2 3}$ \\
$\mathrm{K}(\mathrm{n}=8)$ & $88.75 \pm 33.05$ & $89.63 \pm 37.68$ & $\mathrm{t}=0.123$ \\
& $\mathrm{~F}=0.013$ & $\mathrm{~F}=1.679$ & $\mathrm{p}=0.905$ \\
One Way ANOVA & $\mathrm{p}=0.987$ & $\mathrm{p}=0.208$ & \\
Post Hoc Test & & & \\
\hline
\end{tabular}

individuals butincreased in Pitta Prakritiindividuals. It was found statistically significant on applying nonparametric test for Pitta Prakriti individuals ( $\mathrm{p}=0.045$ ). On applying One Way ANOVA, sweat chloride concentration did not vary statistically significant as per Prakriti (Table 15).
The data shows that mean value of amount of sweat secreted on day first and third in individuals increased on third day as compared to first day but was significant for Pitta Prakriti individuals ( $\mathrm{p}=0.036$ ) (Table 16).

Table 15. Showing the Effect of Sarvanga Ruksha Svedan on sweat chloride (mmol/l) concentration as per Prakriti

\begin{tabular}{cccc}
\multirow{2}{*}{ Prakriti } & \multicolumn{2}{c}{ MEAN \pm SD } & Within the group comparison (nonparametric test) \\
\cline { 2 - 3 } $\mathrm{N}(\mathrm{n}=9)$ & $69.89 \pm 29.02$ & $61.78 \pm 20.41$ & $\mathrm{z}=0.926$ \\
& & & $\mathrm{p}=0.393$ \\
$\mathrm{P}(\mathrm{n}=11)$ & $71.20 \pm 39.81$ & $97.40 \pm 41.94$ & $\mathrm{z}=2.295$ \\
& & & $\mathrm{p}=\mathbf{0 . 0 4 5}$ \\
$\mathrm{K}(\mathrm{n}=8)$ & $81.54 \pm 27.22$ & $73.00 \pm 33.08$ & $\mathrm{z}=1.586$ \\
& $\mathrm{~F}=0.302$ & $\mathrm{~F}=2.803$ & $\mathrm{p}=0.157$ \\
One Way ANOVA & $\mathrm{p}=0.742$ & $\mathrm{p}=0.081$ & \\
Post Hoc Test & - & - & \\
\hline
\end{tabular}

Table 16. Showing the Effect of Sarvanga Ruksha Svedan on amount (in ml) of sweat secreted per Prakriti

\begin{tabular}{cccc}
\hline \multirow{2}{*}{ Prakriti } & \multicolumn{2}{c}{ MEAN \pm SD } & Within the group comparison (paired t-test) \\
\cline { 2 - 3 } $\mathrm{V}(\mathrm{n}=9)$ & $2.21 \pm 0.52$ & $2.51 \pm 0.64$ & $\mathrm{t}=2.404$ \\
$\mathrm{P}(\mathrm{n}=11)$ & $2.48 \pm 0.81$ & $2.73 \pm 0.72$ & $\mathrm{p}=\mathbf{0 . 0 4 3}$ \\
& & $\mathrm{t}=0.696$ \\
$\mathrm{~K}(\mathrm{n}=8)$ & $2.38 \pm 0.63$ & $2.48 \pm 0.62$ & $\mathrm{p}=\mathbf{0 . 0 3 6}$ \\
& $\mathrm{F}=0.383$ & $\mathrm{~F}=0.009$ & $\mathrm{t}=1.871$ \\
One Way ANOVA & $\mathrm{p}=0.686$ & $\mathrm{p}=0.99$ & \\
Post Hoc Test & & & \\
\hline
\end{tabular}




\section{Discussion}

Ayurveda believes that all the diseases are emerged from hypo-functioning of Agni (Mandagni). Svedana karma augments Agni, pacify Vata and Kapha Dosha, improves macro and micro circulation (Srotoshodhak) thus it helps in restoration of physiology and health. Ayurveda has described Svedan (sudation) for detoxification of the body and management of disorders caused by Vata, Kapha Dosha and musculoskeletal disorders. Various methods of sudation have been described as per involvement of Dosha, disorder and season. The two major categories of Svedan are Saagni in which sweating is induced by applying external heat using various means and Niragni means sweating is induced without applying external heat by various measures like exercise, heavy clothing, etc. These two types of Svedan i.e. Saagni and Niragni may be correlated with passive and active heat therapy respectively. In recent past researchers have focused on understanding the mechanism of responses and health benefits of heat therapy in animal models and healthy young individuals. Studies have reported various health benefits of heat therapy which are similar to exercise like anti-inflammatory, vasodilator, improved circulation and metabolism, as well as better glucose control, protein synthesis ${ }^{3}$.

\subsection{Effect of Sarvanga Ruksha Sveda on Physiological Parameters}

Change in mean value of blood pressure after Sravanga Ruksha Svedan Karma was found significant. ( $\mathrm{p}=0.008)$. Systolic blood pressure was reduced after Sravanga Svedan Karma. The change in pressure might have occurred due to fact that heat application leads to vasodilatation, reducing venous return and cardiac output which ultimately results in reduced systolic blood pressure. In 8 weeks' study on Passive heat therapy in sedentary human by Brunt VE et al., 2016 reported that it improves vascular function, flow-mediated dilatation, arterial stiffness and blood pressure. Passive heat therapy improves endothelial function, arterial stiffness and blood pressure in sedentary humans ${ }^{5}$. A study by Tae Won Shin et al., 2018, directs that hot tub bath for 10 minutes lowers the blood pressure in hypertensive patient. So a repeated session of hot tub bath like one to two sessions per week may help those individual to control their blood pressure ${ }^{6}$. This method of hot tub bath may be understood as Avagaha, a type of Svedana Karma. The difference in diastolic blood pressure does not vary significantly however the decrease in the mean value of diastolic blood pressure may be due to decrease blood viscosity by heat and vasodilator effect of Svedana Karma, which leads to decreased peripheral resistance. A study proposed that heat reduces the viscosity of blood which is about $2 \%$ for each 1 degree centigrade rise in temperature. This reduction in viscosity causes decrease in peripheral resistance and increased vasodilatation results in fall of blood pressure ${ }^{7,8}$. The mean value of pulse rate was increased significantly after Sravanga Ruksha Svedan Karma ( $\mathrm{p}=0.001$ ) (Table 1). This effect is justifying the response of body on increase in temperature i.e. increase in temperature leads to increased discharge from SA node of heart thus increasing heart rate and pulse rate ${ }^{8}$. In a study on Haemo-dynamic effects of Sarvang Svedan by S Rastogi et.al 2013, reported an increase in all the measured variables (SBP, DBP, and PR) comparing to the basal values in NTN group IAT and FMAT. This increase was found significant comparing to the basal data in IAT group for all the variables except for DBP. Such an increase of variables was found transient as it reached similar to the basal values in FMAT group 9 . A study by Vienna E. Brunt et. al 2016, on passive heat therapy (thermo neutral water immersion) reported that it improves endothelial function, arterial stiffness and blood pressure in sedentary humans and suggested that passive heat therapy is capable of inducing robust improvements in vascular health, even in sedentary, young (otherwise healthy) individuals. Further they suggested that although exercise is considered a primary treatment for hypertension, exercise training generally has little or no effect on blood pressure in young individuals. Given that heat therapy is capable of lowering blood pressure even in young, normotensive individuals, heat therapy or exercise combined with heat therapy could prove more powerful than exercise alone as a treatment for hypertension ${ }^{5}$. Mean value of temperature was increased significantly on both days after Sarvanga Ruksha Svedan Karma ( $\mathrm{p}=0.001$ ), (Table 1). Study by Wanda Pilch et al., 2014 proposed that there is an increase in core body temperature 
after taking sauna bath that leads to stimulation of sympathetic adrenergic system and enhanced metabolism. The core temperature of body depends on the rate of metabolism, faster the metabolism more will be the temperature with in physiological limit ${ }^{9}$. These observations refer that Sravanga Svedan Karma augments the Agni.

\subsection{Effect of Sarvanga Ruksha Sveda on Hematological Parameters}

The mean value of RBCs has not changed significantly after applying Sravanga Svedan Karma for three days. But from the mean value it can be seen that RBCs count was slightly decreased. For the proper evaluation of effect of Saravanga Ruksha Svedan Karma on RBCs count, the study should be done in large sample size and seven-day regimen of Sravanga Ruksa Svedan Karma should be applied as per textual description. No significant change was observed in mean value of hemoglobin concentration, MVV, MCH, MCHC after applying Sravanga Ruksha Svedan Karma for three days. From the mean value of $\mathrm{Hb}$ it can be seen that hemoglobin concentration has risen which is indicating the increased demand of cellular oxygen due to increased metabolic rate on application of external heat. Since Rakta dhatu is responsible for slight elevation of these values might justify that the Tvak prasadan occurs after application of Sveadan karma ${ }^{1,2}$ (Ch.Su 14/13, S.Chi.32/22) (Table 2). Since Rakta Dhatu is Ashraya of Pitta Dosha (A.H.Su. 12/26-27) ${ }^{3}$ and Svedan causes Pitta Vriddhi. This Pitta Dosha and Rakta Dhatu is responsible for softness and luster of the skin. These parameters like $\mathrm{Hb}, \mathrm{MCH}$ and $\mathrm{MCHC}$ are the indices of Rakta Dhatu, increase in these values is consistent with the Ayurvedic textual description. No significant difference was observed in hematocrite values after Sarvanga Ruksha Svedan Karma for three days. The mean value of WBCs has not changed significantly after applying Sarvanga Ruksha Svedan Karma for three days (Table 2). But from the mean value it can be seen that TLC was increased after Sarvanga Ruksha Svedan Karma. This increase in leukocyte count is suggesting the physiological effect of heat stress on leukocytosis. However neutrophil count was increased significantly $(\mathrm{p}=0.01)$ suggesting the response of body to acute physical heat stress (Table 2), it may be due to enhance secretion of glucocorticoids which causes neutrophilia and decrease the count of other leukocytes ${ }^{11}$.

\subsection{Effect of Sarvanga Ruksha Sveda on Serum Electrolytes}

Significant reduction in serum level of sodium and chloride concentration is justifying the Kledavidhriti function of Sveda (S. Su. 15/8), (A.H.Su. 11/5) 2,3 (Table 3). Mean value of serum level of potassium was not changed significantly after applying Sarvanga Ruksha Svedan for three days but mean value was slightly increased. Charak has described about the composition of human body that major constitution of the body is Ambu (water) i.e. Dehamrasajoambubhavo $\left(\right.$ Ch.Chi.22/16) ${ }^{1}$. The modern science also explains that about $60 \%$ of the adult human bodily content is fluid i.e. a water solution of ions and other substances ${ }^{12}$. There are two sources of fluid for the body, one is Aahara (food) i.e. one among the Chaturvidhaahara is Jala (water). The second is Kleda (moisture), formed inside the body during the process of digestion and metabolism. All the essentials of the Shareera Dhatu are carried by the Dravadhatu continuously by Vyana-Vata (Ch.Chi.15/36) ${ }^{1}$ there by maintaining the normal function of each and every tissue. Sodium and chloride ions are the major constituent of extra cellular fluid. Whenever there is a fluid loss irrespective to any specific condition these two ions are associated with their loss also. As a compensatory mechanism, potassium ion, which is the major constituent of intracellular fluid moves to the extracellular fluid to maintain homeostasis. So this might be the reason of decrease in sodium and chloride level with increment in the level of potassium ${ }^{12}$. However, the changes are within the normal limit when Svedan is performed up to the optimum level i.e Samyak-lakshana, there is no pathological variations in the level of electrolytes. But in some conditions it has been observed that certain electrolytes get increased or decreased indicating to excess removal and lesser retainment of that particular electrolyte. Thus, it could be said that up to the Samyaklakshana of Svedan karma, the levels of the electrolytes remain within the physiological limit. The mean value of serum level of creatinine too was not changed significantly but increase was within the physiological 
limit. It might have occurred due to increased muscular activity under the effect of heat (Table 3).

\subsection{Effect of Sarvanga Ruksha Sveda on Lipid Profile}

The mean values of serum level for all parameters of lipid profiles were not changed significantly after applying Sarvanga Ruksha Svedan for three days. Triglycerides level was decreased while cholesterol, HDL, LDL level was increased. This variation was within the physiological limit and was clinically nonsignificant (Table 3). The study by Dorota Gryka et.al, 2014 revealed that sauna bath reduced the level of LDL and total cholesterol with insignificant increase of HDL-C and transient decline of triacylglycerol's. It shows the positive effect on lipid profile and can be forwarded as an adjuvant treatment for the patient of cardiovascular diseases. It also revealed that there is a significant increase in concentration of free fatty acid which regard to the lipolysis influenced by glucagon, catecholamine, ACTH, cortisol and sympathetic nervous system in force of heat stress $^{13}$. Although a large sample size is required to evaluate the effect of Svedan on these parameters which may be also applied as therapeutic modality in obesity.

\subsection{Effect of Ruksha Sarvanga Svedana as per Prakriti}

The mean value of SBP and DBP was decreased in individuals of all the Prakriti. Significant change was observed for SBP in Pitta Prakriti individuals ( $\mathrm{p}=0.008$ ). The mean value of pulse rate and temperature was significantly increased in all Prakriti individuals. The mean value of duration of the tolerance of heat was increased after Sarvanga Svedan in all Prakriti individual but significantly increased in Pitta Prakriti individual $(\mathrm{p}=0.001)$. It is mentioned in text that Pitta Prakriti individuals are unable to tolerate heat but obtained data in study has shown increased tolerance on day $3^{\text {rd }}$ in present study. The mean value of hemoglobin, platelet, and neutrophil was increased in all Prakriti individuals but in Pitta Prakriti it was found significant. The mean value of sodium and chloride concentration in serum was increased on day 3 as compared to day 1 in all Prakriti individuals. Change in sodium concentration was significant in Vata Prakriti individuals $(\mathrm{p}=0.05)$ while that of chloride concentration was in Pitta Prakriti individuals $(\mathrm{p}=0.035)$. The mean value of volume was increased in all Prakriti individual but was significantly increased in Pitta Prakriti $(\mathrm{p}=0.036)$ and Vata Prakriti $(\mathrm{p}=0.043)$. Charak has described that the Drava property of Pitta is responsible for the excessive exertion of sweat, urine, and stool in people of Pitta Prakriti $^{1}$ (Ch.Vi.8/67). On sweat analysis, mean value of sweat LDH was decreased in $3^{\text {rd }}$ day as compared to $1^{\text {st }}$ day, in all the subjects except to Kapha Prakriti individuals. The changes were found significant for Vata Prakriti $(\mathrm{p}=0.024)$. The mean value of sweat sodium was significantly increased in Pitta Prakriti individual $(\mathrm{p}=0.023)$. Mean value of sweat creatinine and sweat chloride was significantly increased in Pitta Prakriti individuals.

\section{Conclusion}

Svedana is used as a treatment modality mainly as Purvkarma in Ayurveda for management of disorders. No sustained safe period of Svedan is determined, only certain characteristics have been explained. In this study, we tried to establish and found a safe period for swedan in individuals of different Prakriti i.e. duration of approximately less than 25 minutes, 24 minutes and 28 minutes for Vata, Pitta and Kapha Prakriti individual in winter season. Results have shown that Swedan for such period in respective Prakriti will not produce any undue effects. The physiological, hematological and biochemical parameters, have shown changes as per Prakiriti but were within the physiological ranges. Significant decrease is observed in SPB in Pitta prakriti and Serum sodium in Vata Prakriti and decreased TG were found. Thus, the present study has substantiated the views of Ayurveda scholars that the sweat is a media for removal of metabolic waste products and further studies could be performed on different methods of Svedan Karma may produces different effects on sweat composition and body.

\section{Limitations of Study}

This study was conducted on small sample and purposive sampling technique was used. Results were 
not evaluated in accordance to sex. Svedana is given for shorter period for three days. Further there is a need of robust documentation and through series of investigation to potentiate the outcome.

\section{Acknowledgement}

The authors acknowledge the support and facilities they received during the present work from the Department of Kriya Sharir, Department of Kaya Chikitsa, Panchakarma Unit, Faculty of Ayurveda, CCI lab S S hospital, IMS, Banaras Hindu University, Varanasi.

\section{References}

1. Sharma RK, Das B. Charak Samhita of Agnivesh (Reprint edition). Varanasi: Chaukhambha Sanskrit Series Office; 2015.

2. Shrikantha KRM. SushrutaSamhita of Sushruta (Reprint edition). Varanasi: .Chaukhambha Orientalia; 2014.

3. Shrikantha KRM. Ashtanga Hridayam of Vagbhatta (10th edition). Chaukhambha Krishanadas Academy; 2014.

4. Thomas KN. Harnessing heat for health: A clinical application of heat stress. Temperature (Austin). 2017; 4(3):208-10. https://doi.org/10.1080/23328940.2017.13173 79. PMid:28944265 PMCid:PMC5605166

5. Brunt VE, Howard MJ, Francisco MA, Ely BR, Minson CT. Passive heat therapy improves endothelial function, arterial stiffness and blood pressure in sedentary humans. Journal of
Physiology. 2016; 594(18):5329-42.https://doi.org/10.1113/ JP272453. PMid:27270841 PMCid:PMC5023696

6. Shin TW, Wilson M, Wilson TW. Are hot tubs safe for people with treated hypertension? CMAJ. 2003; 169(12):1265-8.

7. Reddy LP. Fundamentals of Medical Physiology, 3rd edition, Paras Medical Publisher; 2003. Chapter 5, p. 243.

8. Guyton and Hall. Textbook of Medical Physiology (Reprint edition). A south Asian edition; 2014. p. 219.

9. Rastogi S, Chiappelli F. Hemodynamic effects of Sarvanga Swedana (Ayurvedic passive heat therapy): A pilot observational study. Ayu. 2013; 34(2):154-9. https:// doi.org/10.4103/0974-8520.119669. PMid:24250123 PMCid:PMC3821243

10. Pilch W, Szygula Z, Tyka A, Pałka T, Lech G, Cisoń T, et al. Effect of 30-minute sauna sessions on lipid profile in young women. Medicina Sportiva. 2014; 18:165-71. https://doi. org/10.5604/17342260.1133107

11. Jain AK. Textbook of Physiology. (Revised edition). Volume 2, Avichal Publishing Company; 2009. p. 741

12. Guyton and Hall. Textbook of Medical Physiology (Reprint edition). A South Asian edition; 2014. p. 3.

13. Gryka D, Pilch W, Szarek M, Szygula Z, Tota L. The effect of sauna bathing on lipid profile in young, physically active, male subjects. International Journal of Occupational Medicine and Environmental Health. 2014; 27(4): 608-18. https://doi.org/10.2478/s13382-014-0281-9. PMid:25001587 